\title{
Analysis of the nonlocal wave propagation problem with volume constraints
}

\author{
Ait Bella F. Z. ${ }^{1}$, El Rhabi M. ${ }^{2}$, Hakim A. ${ }^{1}$, Laghrib A. ${ }^{3}$ \\ ${ }^{1}$ Cadi Ayyad University, \\ Abdelkrim Khattabi Avenue, Marrakesh, 40000, Morocco \\ ${ }^{2}$ Institut Polytechnique UniLaSalle, \\ 3 rue du Tronquet, Mont-Saint-Aignan, 76130, France \\ ${ }^{3}$ Sultan Moulay Slimane University, \\ Avenue Ibn Khaldoun, Béni-Mellal, 23000, Morocco
}

(Received 12 June 2020; Revised 24 July 2020; Accepted 27 July 2020)

\begin{abstract}
In the current paper, we develop a nonlocal propagation model, which describes the diffusion wave process. The main motivation of this work is to apply the nonlocal vector calculus, introduced and developed by Du et al. [1] to such hyperbolic problem. Moreover, based on some density arguments, some a priori estimates and using the Galerkin approach, we prove existence and uniqueness of a weak solution to the nonlocal wave equation widely adopted in various applications.
\end{abstract}

Keywords: Galerkin approximation, nonlocal operators, nonlocal vector calculus, volume constrained problems, wave equation.

\section{Introduction}

From a physical standpoint, nonlocal approaches play a vital role in characterizing many natural phenomena. The concern for nonlocal methods is motivated by the ability of these approaches to capture with rigorous accuracy the effects that are difficult to describe by local models. Nonlocal functionals, nonlocal operators and nonlocal problems defined in nonlocal function spaces, have gradually attracted the mathematical community's attention by its theoretical value, as for its concrete real-world applications. This type of model occurs in a quite natural way in many different contexts, such as, among others, image restoration [2-5], phase transition [6,7], machine learning [8] and obstacle problem [3].

In a major advance in 2013, Du et al. [1] offered a collection of relevant results to better understand and analyze nonlocal problems. The suggested nonlocal vector calculus defines nonlocal fluxes, nonlocal analogues of the gradient, divergence, and curl operators, and presents some basic nonlocal integral theorems that mimic the classical integral theorems of the vector calculus for differential operators, the authors have also provided connection between the nonlocal operators and their usual differential counterparts in a distributional sense then in a weak sense by introducing nonlocal weighted operators.

The present paper was motivated by [9], where the authors threw light on the analogy between nonlocal and local diffusion problems with a convincing explanation of the usefulness, in the nonlocal case, of volume constraints which represent the nonlocal analogue of the boundary conditions of the classical theory. Our purpose is to discuss the well-posedness of a hyperbolic problem considering a nonlocal diffusion operator instead of the Laplacian operator. Furthermore, the study of the eigenvalues problem corresponding to the nonlocal Dirichlet problem is carried out.

The paper is divided into two main sections. The first part gives a brief overview of the basic concepts of the nonlocal vector calculus and emphasises the existence of an orthogonal basis of eigenfunctions associated to the considered nonlocal operator. In the second part, we formulate the nonlocal wave equation and exploit the Galerkin method to prove existence and uniqueness of weak solution to the nonlocal hyperbolic problem. 


\section{Statement of the elliptic nonlocal problem}

In the present section, we give the position of the elliptic volume constrained problem and present the energy spaces needed to study the nonlocal problem:

$$
\left\{\begin{array}{lll}
\mathcal{D}\left(\xi \cdot \mathcal{D}^{*}(u)\right)=f & \text { on } & \Omega \\
u=0 & \text { on } & \Omega_{I}
\end{array}\right.
$$

Where $\Omega$ is an open and bounded subset of $\mathbb{R}^{n}$ with piecewise smooth boundary and satisfies the interior cone condition, $\xi$ is a symmetric second-order tensor, $f \in L^{2}(\Omega)$ is a given function and we denote by " $u . v$ " the tensor-vector product or the inner product of two vectors $u$ and $v$.

Given a vector function $\nu(x, y): \mathbb{R}^{n} \times \mathbb{R}^{n} \rightarrow \mathbb{R}^{k}$ and an antisymmetric vector function $\alpha(x, y): \mathbb{R}^{n} \times$ $\mathbb{R}^{n} \rightarrow \mathbb{R}^{k}$, the action of the nonlocal divergence operator $\mathcal{D}$ on $\nu$ is defined as

$$
\mathcal{D}(\nu)(x):=\int_{\mathbb{R}^{n}}(\nu(x, y)+\nu(y, x)) \cdot \alpha(x, y) d y \quad \text { for } \quad x \in \mathbb{R}^{n} .
$$

Given a scalar function $u(x): \mathbb{R}^{n} \rightarrow \mathbb{R}$, the adjoint of $\mathcal{D}$ is the operator $\mathcal{D}^{*}$ whose action on $u$ is given by

$$
\mathcal{D}^{*}(u)(x, y)=-(u(y)-u(x)) \alpha(x, y) \quad \text { for } \quad x, y \in \mathbb{R}^{n} .
$$

The operator $-\mathcal{D}^{*}$ is considered as a nonlocal gradient, also,

$$
\mathcal{D}\left(\xi \cdot \mathcal{D}^{*} u\right)(x)=-2 \int_{\mathbb{R}^{n}}(u(y)-u(x)) \alpha(x, y) \cdot(\xi(x, y) \cdot \alpha(x, y)) d y .
$$

Given two positive constants $\gamma_{0}$ and $\varepsilon$, we first assume that the symmetric kernel

$$
\gamma(x, y)=\alpha(x, y) \cdot(\xi(x, y) \cdot \alpha(x, y))
$$

satisfies, for all $x \in \Omega \cup \Omega_{I}$

1. $\gamma(x, y) \geqslant 0 \quad \forall y \in B_{\varepsilon}(x)$;

2. $\gamma(x, y) \geqslant \gamma_{0}>0 \quad \forall y \in B_{\varepsilon / 2}(x)$

3. $\gamma(x, y)=0 \quad \forall y \in\left(\Omega \cup \Omega_{I}\right) \backslash B_{\varepsilon}(x)$, where $B_{\varepsilon}(x):=\left\{y \in \Omega \cup \Omega_{I}:|y-x| \leqslant \varepsilon\right\}$;

4. There exist $s \in(0,1)$ and positive constants $\gamma_{*}$ and $\gamma^{*}$ such that, for all $x \in \Omega$

$$
\frac{\gamma_{*}}{|y-x|^{n+2 s}} \leqslant \gamma(x, y) \leqslant \frac{\gamma^{*}}{|y-x|^{n+2 s}} \quad \text { for } \quad y \in B_{\varepsilon}(x) .
$$

Let us also recall the definition of the interaction domain corresponding to $\Omega$ :

$$
\Omega_{I}:=\left\{y \in \mathbb{R}^{n} \backslash \Omega: \alpha(x, y) \neq 0 \quad \text { for some } \quad x \in \Omega\right\} .
$$

To investigate the problem (1), the following nonlocal energy space will be used constantly [9]. We adopt:

$$
V\left(\Omega \cup \Omega_{I}\right):=\left\{u \in L^{2}\left(\Omega \cup \Omega_{I}\right):\||u|\|<\infty\right\}
$$

equipped with the nonlocal energy norm

$$
\|\mid u\|:=\left(\frac{1}{2} \int_{\Omega \cup \Omega_{I}} \int_{\Omega \cup \Omega_{I}} \mathcal{D}^{*}(u) \cdot\left(\xi \cdot \mathcal{D}^{*}(u)\right) d y d x\right)^{\frac{1}{2}},
$$

we then introduce the nonlocal volume constrained energy space [9]:

$$
V_{c}\left(\Omega \cup \Omega_{I}\right):=\left\{u \in V\left(\Omega \cup \Omega_{I}\right): u=0 \quad \text { in } \quad \Omega_{I}\right\},
$$

Mathematical Modeling and Computing, Vol. 7, No. 2, pp. 334-344 (2020) 
the norm

$$
|| f\left|\|_{V_{c}^{*}\left(\Omega \cup \Omega_{I}\right)}:=\sup _{\substack{\varphi \in V_{c}\left(\Omega \cup \Omega_{I}\right) \\\|\varphi\| \| \leqslant 1}}\right|\langle f, \varphi\rangle_{V_{c}^{*}\left(\Omega \cup \Omega_{I}\right), V_{c}\left(\Omega \cup \Omega_{I}\right)} \mid
$$

denotes the norm for the dual space $V_{c}^{*}\left(\Omega \cup \Omega_{I}\right)$ of $V_{c}\left(\Omega \cup \Omega_{I}\right)$.

Next, using the nonlocal Green's first identities [1], we state the following definition.

Definition 1. We say that $u \in V_{c}\left(\Omega \cup \Omega_{I}\right)$ is a weak solution of the nonlocal elliptic problem (1) if

$$
\int_{\Omega \cup \Omega_{I}} \int_{\Omega \cup \Omega_{I}} \mathcal{D}^{*}(u) \cdot\left(\xi \cdot \mathcal{D}^{*}(\varphi)\right) d y d x=\int_{\Omega} f \varphi d x \quad \forall \varphi \in V_{c}\left(\Omega \cup \Omega_{I}\right) .
$$

Then, according to the definition of the nonlocal energy norm (6), we immediately announce the following theorem:

Theorem 1. There exist two constants $M_{1}, M_{2}>0$ such that

$$
\left|\int_{\Omega \cup \Omega_{I}} \int_{\Omega \cup \Omega_{I}} \mathcal{D}^{*}(u) \cdot\left(\xi \cdot \mathcal{D}^{*}(\varphi)\right) d y d x\right| \leqslant M_{1}|||u||||||\varphi| \| \mid
$$

and

$$
M_{2}|\|u\||^{2} \leqslant \int_{\Omega \cup \Omega_{I}} \int_{\Omega \cup \Omega_{I}} \mathcal{D}^{*}(u) \cdot\left(\xi \cdot \mathcal{D}^{*}(u)\right) d y d x
$$

for all $u, \varphi \in V_{c}\left(\Omega \cup \Omega_{I}\right)$

Theorem 2. For each $f \in L^{2}(\Omega)$, there exists a unique weak solution $u \in V_{c}\left(\Omega \cup \Omega_{I}\right)$ of the nonlocal elliptic problem (1).

Proof. Using the previous theorem (1), we obtain the result of existence and uniqueness via a direct application of the Lax-Milgram theorem.

\subsection{The nonlocal Dirichlet eigenvalues problem}

In this subsection, we focus our attention on seeking the set of numbers $\mu$ such that the following eigenvalues problem (10) corresponding to the Dirichlet nonlocal problem (1):

$$
\int_{\Omega \cup \Omega_{I}} \int_{\Omega \cup \Omega_{I}} \mathcal{D}^{*}(u) \cdot\left(\xi \cdot \mathcal{D}^{*}(\varphi)\right) d y d x=\mu \int_{\Omega} u \varphi d x \quad \forall \varphi \in V_{c}\left(\Omega \cup \Omega_{I}\right),
$$

has a solution $u \in V_{c}\left(\Omega \cup \Omega_{I}\right)$.

We state the following result.

Theorem 3. 1) Each eigenvalue of the problem (10) is real.

2) If we repeat each eigenvalue according to its multiplicity, we have that the set $\Sigma$ of the eigenvalues of the operator $\mathcal{D}\left(\xi . \mathcal{D}^{*}().\right)$ is as follows:

$$
\Sigma=\left(\mu_{j}\right)_{j \geqslant 1},
$$

where $0<\mu_{1} \leqslant \mu_{2} \leqslant \ldots \leqslant \mu_{n} \leqslant \ldots$ and $\mu_{j} \underset{j \rightarrow \infty}{\longrightarrow} \infty$.

3) There exists an orthonormal basis $\left(v_{j}\right)_{j \geqslant 1}$ of $L^{2}\left(\Omega \cup \Omega_{I}\right)$, where $v_{j} \in V_{c}\left(\Omega \cup \Omega_{I}\right)$ is an eigenvector corresponding to $\mu_{j}$ for $j \geqslant 1$.

Proof. Let $K$ be the mapping

$$
\begin{aligned}
K: L^{2}\left(\Omega \cup \Omega_{I}\right) & \rightarrow V_{c}\left(\Omega \cup \Omega_{I}\right), \\
f & \mapsto u_{f},
\end{aligned}
$$

Mathematical Modeling and Computing, Vol. 7, No. 2, pp. 334-344 (2020) 
where $u$ is the unique solution of (1) given by Theorem (2).

We claim that the operator $K$ is bounded, indeed:

$$
\begin{aligned}
\|u\| \|^{2} & =\int_{\Omega \cup \Omega_{I}} \int_{\Omega \cup \Omega_{I}} \mathcal{D}^{*}(u) \cdot\left(\xi \cdot \mathcal{D}^{*}(u)\right) d y d x \\
& \leqslant\|f\|_{L^{2}(\Omega)}\|u\|_{L^{2}(\Omega)},
\end{aligned}
$$

according to the nonlocal Poincare inequality [9], there exists a positive constant $C$ such that:

$$
\|K f \mid\| \leqslant\|f\|_{L^{2}\left(\Omega \cup \Omega_{I}\right)}
$$

since the embedding

$$
\begin{aligned}
I: V_{c}\left(\Omega \cup \Omega_{I}\right) & \rightarrow L^{2}\left(\Omega \cup \Omega_{I}\right), \\
u & \mapsto u
\end{aligned}
$$

is compact [9], we directly deduce that the mapping

$$
\begin{aligned}
I \circ K: L^{2}\left(\Omega \cup \Omega_{I}\right) & \rightarrow L^{2}\left(\Omega \cup \Omega_{I}\right), \\
f & \mapsto u_{f}
\end{aligned}
$$

is linear and compact.

On the other hand, if $w$ is the unique solution of the problem:

$$
\left\{\begin{array}{lll}
D\left(\xi \cdot D^{*}(w)\right)=f & \text { on } & \Omega \\
w=0 & \text { on } & \Omega_{I}
\end{array}\right.
$$

and $v$ if the solution of:

$$
\left\{\begin{array}{lll}
D\left(\xi \cdot D^{*}(v)\right)=g & \text { on } & \Omega \\
v=0 & \text { on } & \Omega_{I}
\end{array}\right.
$$

where $f, g \in L^{2}\left(\Omega \cup \Omega_{I}\right)$. We have:

$$
\begin{aligned}
& ((I \circ K) f, g)_{L^{2}\left(\Omega \cup \Omega_{I}\right)}=\int_{\Omega} w g d x=\int_{\Omega \cup \Omega_{I}} \int_{\Omega \cup \Omega_{I}} \mathcal{D}^{*}(v) \cdot\left(\xi \cdot \mathcal{D}^{*}(w)\right) d y d x, \\
& ((I \circ K) g, f)_{L^{2}\left(\Omega \cup \Omega_{I}\right)}=\int_{\Omega} v f d x=\int_{\Omega \cup \Omega_{I}} \int_{\Omega \cup \Omega_{I}} \mathcal{D}^{*}(w) \cdot\left(\xi \cdot \mathcal{D}^{*}(v)\right) d y d x,
\end{aligned}
$$

which prove that the operator $I \circ K$ is symmetric. In addition:

$$
((I \circ K) f, f)_{L^{2}\left(\Omega \cup \Omega_{I}\right)}=\int_{\Omega \cup \Omega_{I}} \int_{\Omega \cup \Omega_{I}} \mathcal{D}^{*}(w) \cdot\left(\xi \cdot \mathcal{D}^{*}(w)\right) d y d x \geqslant 0 .
$$

We apply the theory of compact and symmetric operators from [13] to conclude the existence of real eigenvalues of $I \circ K$, and that the corresponding eigenvectors $\left(v_{j}\right)_{j \geqslant 1}$ form a complete orthonormal system in $L^{2}\left(\Omega \cup \Omega_{I}\right)$.

To conclude the proof, notice that:

$$
(I \circ K) v=\lambda v \text { is equivalent to } \mathcal{D}\left(\xi \cdot \mathcal{D}^{*}(v)\right)=\frac{1}{\lambda} v=\mu v .
$$

Theorem 4. Let $\left(v_{j}\right)_{j \geqslant 1}$ be the eigenvectors corresponding to $\left(\mu_{j}\right)_{j \geqslant 1}$ given by Theorem 3 , then $\left(v_{j}\right)_{j \geqslant 1}$ forms an orthogonal basis of $V_{c}\left(\Omega \cup \Omega_{I}\right)$. 
Proof. The orthogonality of the eigenvectors follows from:

$$
\begin{aligned}
\int_{\Omega \cup \Omega_{I}} \int_{\Omega \cup \Omega_{I}} \mathcal{D}^{*}\left(v_{j}\right) \cdot\left(\xi \cdot \mathcal{D}^{*}\left(v_{k}\right)\right) d y d x & =\mu_{j}\left(v_{j}, v_{k}\right)_{L^{2}(\Omega)} \\
& =\mu_{j} \delta_{i, j} .
\end{aligned}
$$

On the other hand, for each $u \in V_{c}\left(\Omega \cup \Omega_{I}\right)$ we have:

$$
\begin{aligned}
u & =\sum_{j \geqslant 1}\left(u, v_{j}\right)_{L^{2}\left(\Omega \cup \Omega_{I}\right)} v_{j} \\
& =\sum_{j \geqslant 1} \frac{\int_{\Omega \cup \Omega_{I}} \int_{\Omega \cup \Omega_{I}} \mathcal{D}^{*}(u) \cdot\left(\xi \cdot \mathcal{D}^{*}\left(v_{j}\right)\right) d y d x}{\mu_{j}} v_{j} \\
& =\sum_{j \geqslant 1} \frac{\int_{\Omega \cup \Omega_{I}} \int_{\Omega \cup \Omega_{I}} \mathcal{D}^{*}(u) \cdot\left(\xi \cdot \mathcal{D}^{*}\left(v_{j}\right)\right) d y d x}{\left\|v_{j}\right\|^{2}} v_{j},
\end{aligned}
$$

which concludes the proof.

Since $\left(v_{j}\right)_{j \geqslant 1}$ is an orthogonal basis of $V_{c}\left(\Omega \cup \Omega_{I}\right)$ for any $j \in \mathbb{N}$, we can define the orthogonal projection on the j-dimensional subspace of $V_{c}\left(\Omega \cup \Omega_{I}\right)$ spanned by $v_{1}, v_{2}, \ldots, v_{j}$.

Proposition 5. Let $P_{n}, Q_{n}$ be the orthogonal projections defined, for all $n \in \mathbb{N}$, by:

$$
\begin{aligned}
P_{n}(u) & :=\sum_{j=1}^{n}\left(u, v_{j}\right)_{L^{2}\left(\Omega \cup \Omega_{I}\right)} v_{j} \quad \forall u \in L^{2}\left(\Omega \cup \Omega_{I}\right), \\
Q_{n}(u) & :=\sum_{j=1}^{n} \frac{\int_{\Omega \cup \Omega_{I}} \int_{\Omega \cup \Omega_{I}} \mathcal{D}^{*}(u) \cdot\left(\xi \cdot \mathcal{D}^{*}\left(v_{j}\right)\right) d y d x}{\left.\left\|v_{j}\right\|\right|^{2}} v_{j} \quad \forall u \in V_{c}\left(\Omega \cup \Omega_{I}\right) .
\end{aligned}
$$

Then

$$
P_{n} u \stackrel{L^{2}\left(\Omega \cup \Omega_{I}\right)}{\longrightarrow} u \quad \forall u \in L^{2}\left(\Omega \cup \Omega_{I}\right) .
$$

Then

$$
Q_{n} u \stackrel{V_{c}\left(\Omega \cup \Omega_{I}\right)}{\longrightarrow} u \quad \forall u \in V_{c}\left(\Omega \cup \Omega_{I}\right)
$$

These convergences come simply from the following result.

Proposition 6. Let $P_{n}, Q_{n}$ be the orthogonal projections defined by definitions (12) and (13), then:

$$
\left\|P_{n}\right\|_{\mathcal{L}\left(L^{2}\left(\Omega \cup \Omega_{I}\right), L^{2}\left(\Omega \cup \Omega_{I}\right)\right)}=\left\|Q_{n}\right\|_{\mathcal{L}\left(V_{c}\left(\Omega \cup \Omega_{I}\right), V_{c}\left(\Omega \cup \Omega_{I}\right)\right)}=1 .
$$

If we set

$$
P_{n}(u)=\sum_{j=1}^{n}\left\langle u, v_{j}\right\rangle_{V_{c}^{*}\left(\Omega \cup \Omega_{I}\right), V_{c}\left(\Omega \cup \Omega_{I}\right)} v_{j} \quad \forall u \in V_{c}^{*}\left(\Omega \cup \Omega_{I}\right),
$$

then

$$
\left\|P_{n}\right\|_{\mathcal{L}\left(V_{c}^{*}\left(\Omega \cup \Omega_{I}\right), V_{c}^{*}\left(\Omega \cup \Omega_{I}\right)\right)}=1 .
$$

Proof. Let $u \in L^{2}\left(\Omega \cup \Omega_{I}\right)$, then

$$
\|u\|_{L^{2}\left(\Omega \cup \Omega_{I}\right)}^{2}=\lim _{n \rightarrow \infty} \sum_{j=1}^{n}\left(u, v_{j}\right)_{L^{2}\left(\Omega \cup \Omega_{I}\right)}^{2}=\lim _{n \rightarrow \infty}\left\|P_{n}(u)\right\|_{L^{2}\left(\Omega \cup \Omega_{I}\right)}^{2}
$$

Mathematical Modeling and Computing, Vol. 7, No. 2, pp. 334-344 (2020) 
subsequently

$$
\left\|P_{n}\right\|_{\mathcal{L}\left(L^{2}\left(\Omega \cup \Omega_{I}\right), L^{2}\left(\Omega \cup \Omega_{I}\right)\right)}=\sup _{\substack{u \in L^{2}\left(\Omega \cup \Omega_{I}\right) \\ u \neq 0}} \frac{\left\|P_{n}(u)\right\|_{L^{2}\left(\Omega \cup \Omega_{I}\right)}}{\|u\|_{L^{2}\left(\Omega \cup \Omega_{I}\right)}} \leqslant 1
$$

to conclude the proof, note that: $P_{n}\left(v_{j}\right)=v_{j}$.

Secondly, we have, for $u \in V_{c}\left(\Omega \cup \Omega_{I}\right)$ :

$$
\begin{aligned}
Q_{n}(u) & =\sum_{j=1}^{n} \frac{\int_{\Omega \cup \Omega_{I}} \int_{\Omega \cup \Omega_{I}} \mathcal{D}^{*}(u) \cdot\left(\xi \cdot \mathcal{D}^{*}\left(v_{j}\right)\right) d y d x}{\left.\left\|v_{j}\right\|\right|^{2}} v_{j} \\
& =\sum_{j=1}^{n}\left(u, v_{j}\right)_{L^{2}\left(\Omega \cup \Omega_{I}\right)} v_{j},
\end{aligned}
$$

therefore

$$
\begin{aligned}
\left\|Q_{n}(u)\right\| & =\sum_{j=1}^{n}\left(u, v_{j}\right)_{L^{2}\left(\Omega \cup \Omega_{I}\right)}^{2} \frac{\int_{\Omega \cup \Omega_{I}} \int_{\Omega \cup \Omega_{I}} \mathcal{D}^{*}\left(v_{j}\right) \cdot\left(\xi \cdot \mathcal{D}^{*}\left(v_{j}\right)\right) d y d x}{\left\|\left|v_{j} \|\right|^{2}\right.} v_{j} \\
& \leqslant \sum_{j=1}^{\infty}\left(u, v_{j}\right)_{L^{2}\left(\Omega \cup \Omega_{I}\right)}^{2}\|\| v_{j} \|^{2} \\
& \leqslant\|\| u\|\|^{2},
\end{aligned}
$$

and as $Q_{n}\left(v_{j}\right)=v_{j}$, we claim that $\|\left. Q_{n}\right|_{\mathcal{L}\left(V_{c}\left(\Omega \cup \Omega_{I}\right), V_{c}\left(\Omega \cup \Omega_{I}\right)\right)}=1$.

Furthermore, if we extend the projection $P_{n}$ to $V_{c}^{*}\left(\Omega \cup \Omega_{I}\right)$ we obtain:

$$
\begin{aligned}
\left|\left\langle P_{n}(u), \varphi\right\rangle_{V_{c}^{*}\left(\Omega \cup \Omega_{I}\right), V_{c}\left(\Omega \cup \Omega_{I}\right)}\right| & =\left|\sum_{j=1}^{n}\left\langle u, v_{j}\right\rangle_{V_{c}^{*}, V_{c}}\left(\varphi, v_{j}\right)_{L^{2}\left(\Omega \cup \Omega_{I}\right)}\right| \\
& =\left|\left\langle u, P_{n}(\varphi)\right\rangle_{V_{c}^{*}\left(\Omega \cup \Omega_{I}\right), V_{c}\left(\Omega \cup \Omega_{I}\right)}\right| \\
& \leqslant\left.|| u\right|_{V_{c}^{*}\left(\Omega \cup \Omega_{I}\right)}|||\varphi| \|
\end{aligned}
$$

hence

$$
\left.\left\|\left.P_{n}(u)\right|_{V_{c}^{*}\left(\Omega \cup \Omega_{I}\right)} \leqslant\right\| u\right|_{V_{c}^{*}\left(\Omega \cup \Omega_{I}\right)} .
$$

\section{The nonlocal wave equation}

\section{1. statement of the problem}

We denote by $\Omega$ an open set of $\mathbb{R}^{n}$ and by $\Omega_{I}$ its corresponding interaction domain. We will always assume that $\Omega$ and $\Omega_{I}$ are bounded with piecewise smooth boundary and satisfy the interior cone condition.

The example of nonlocal hyperbolic equation that we consider is the following: we seek a real valued function $u=u(x, t), x \in \Omega, t \in] 0, T]$, solution to

$$
\begin{cases}u^{\prime \prime}+\mathcal{D}\left(\xi \cdot \mathcal{D}^{*}(u)\right)=f & \text { in } \Omega \times] 0, T], \\ u=0 & \text { in } \left.\left.\Omega_{I} \times\right] 0, T\right], \\ u(x, 0)=g(x) & \text { in } \Omega, \\ u^{\prime}(x, 0)=h(x) & \text { in } \Omega .\end{cases}
$$


Where $\mathcal{D}, \mathcal{D}^{*}$ are, respectively, the nonlocal divergence (2) and the nonlocal gradient $(3), \xi(x, y)$ denotes a symmetric, positive definite second order tensor having elements that are symmetric functions of $x$ and $y$ and $f: \Omega \times] 0, T[\rightarrow \mathbb{R}, g, h: \Omega \rightarrow \mathbb{R}$ are given.

First, we specify in which sense we want to solve the problem (14).

Definition 2. If $f \in L^{2}\left(0, T ; L^{2}(\Omega)\right), g \in V_{c}\left(\Omega \cup \Omega_{I}\right)$ and $h \in L^{2}\left(\Omega \cup \Omega_{I}\right)$ we say a function $u \in$ $L^{2}\left(0, T ; V_{c}\left(\Omega \cup \Omega_{I}\right)\right)$ with $u^{\prime} \in L^{2}\left(0, T ; L^{2}\left(\Omega \cup \Omega_{I}\right)\right)$ and $u^{\prime \prime} \in L^{2}\left(0, T ; V_{c}^{*}\left(\Omega \cup \Omega_{I}\right)\right)$ is a weak solution of the nonlocal constrained problem (14) if

$$
\left\langle u^{\prime \prime}, \varphi\right\rangle_{V_{c}^{*}\left(\Omega \cup \Omega_{I}\right), V_{c}\left(\Omega \cup \Omega_{I}\right)}+\int_{\Omega \cup \Omega_{I}} \int_{\Omega \cup \Omega_{I}} \mathcal{D}^{*}(u) \cdot\left(\xi \cdot \mathcal{D}^{*}(\varphi)\right) d y d x=\int_{\Omega} f \varphi d x
$$

$\forall \varphi \in V_{c}\left(\Omega \cup \Omega_{I}\right)$ and a.e. $0 \leqslant t \leqslant T$, with $u(0)=g, u^{\prime}(0)=h$.

Remark 1. Defining: $L_{c}^{2}\left(\Omega \cup \Omega_{I}\right):=\left\{u \in L^{2}\left(\Omega \cup \Omega_{I}\right): u=0\right.$ in $\left.\Omega_{I}\right\}$, the embedding $V_{c}\left(\Omega \cup \Omega_{I}\right) \hookrightarrow$ $L_{c}^{2}\left(\Omega \cup \Omega_{I}\right)$ is dense and continuous, and $L_{c}^{2}\left(\Omega \cup \Omega_{I}\right)$ is a separable Hilbert space since it is a closed subspace of $L^{2}\left(\Omega \cup \Omega_{I}\right)$ and according to Du et al. [9], $V_{c}\left(\Omega \cup \Omega_{I}\right)$ is a Hilbert space which is a subset of $L_{c}^{2}\left(\Omega \cup \Omega_{I}\right)$ with continuous embedding, hence $V_{c}\left(\Omega \cup \Omega_{I}\right)$ is separable. Which ultimately leads to assert that

$$
V_{c}\left(\Omega \cup \Omega_{I}\right) \hookrightarrow L_{c}^{2}\left(\Omega \cup \Omega_{I}\right) \hookrightarrow V_{c}^{*}\left(\Omega \cup \Omega_{I}\right)
$$

is an evolution triple. Consequently, $L_{c}^{2}\left(\Omega \cup \Omega_{I}\right)$ is dense in $V_{c}^{*}\left(\Omega \cup \Omega_{I}\right)$ [10].

Since $u \in S_{2} \subset S$ and $u^{\prime} \in S_{1}$, where

$$
\begin{aligned}
S & =\left\{u \mid u \in L^{2}\left(0, T ; V_{c}\left(\Omega \cup \Omega_{I}\right)\right), u^{\prime} \in L^{2}\left(0, T ; V_{c}^{*}\left(\Omega \cup \Omega_{I}\right)\right)\right\}, \\
S_{1} & =\left\{u \mid u \in L^{2}\left(0, T ; L_{c}^{2}\left(\Omega \cup \Omega_{I}\right)\right), u^{\prime} \in L^{2}\left(0, T ; V_{c}^{*}\left(\Omega \cup \Omega_{I}\right)\right)\right\}, \\
S_{2} & =\left\{u \mid u \in L^{2}\left(0, T ; V_{c}\left(\Omega \cup \Omega_{I}\right)\right), u^{\prime} \in L^{2}\left(0, T ; L_{c}^{2}\left(\Omega \cup \Omega_{I}\right)\right)\right\} .
\end{aligned}
$$

Proposition 23.23. in [10] implies that:

$$
u \in \mathcal{C}\left([0, T] ; L_{c}^{2}\left(\Omega \cup \Omega_{I}\right)\right), \quad u^{\prime} \in \mathcal{C}\left([0, T] ; V_{c}^{*}\left(\Omega \cup \Omega_{I}\right)\right) .
$$

\subsection{Galerkin approximation}

Let $\left(v_{j}\right)_{j \geqslant 1}$ be the eigenvectors corresponding to the eigenvalues $\left(\lambda_{j}\right)_{j \geqslant 1}$ of the problem (10), given by Theorem 3.

For a fixed $n \geqslant 1$, we are looking for a function $u_{n}:[0, T] \rightarrow V_{c}\left(\Omega \cup \Omega_{I}\right)$ of the form

$$
u_{n}(t):=\sum_{j=1}^{n} p_{j n}(t) v_{j},
$$

such that

$$
\left\{\begin{array}{l}
\text { For } \quad j=1, \ldots, n \\
\left\langle u_{n}^{\prime \prime}, v_{j}\right\rangle_{V_{c}^{*}\left(\Omega \cup \Omega_{I}\right), V_{c}\left(\Omega \cup \Omega_{I}\right)}+\int_{\Omega \cup \Omega_{I}} \int_{\Omega \cup \Omega_{I}} \mathcal{D}^{*}\left(u_{n}\right) \cdot\left(\xi \cdot \mathcal{D}^{*}\left(v_{j}\right)\right) d y d x=\int_{\Omega} f_{n} v_{j} d x, \\
u_{n}(0)=\sum_{j=1}^{n}\left(g, v_{j}\right)_{L^{2}(\Omega)} v_{j}, \\
u_{n}^{\prime}(0)=\sum_{j=1}^{n}\left(h, v_{j}\right)_{L^{2}(\Omega)} v_{j},
\end{array}\right.
$$

where $\left(f_{n}\right)_{n} \in \mathcal{D}(\Omega \times(0, T))$ such that $f_{n} \stackrel{L^{2}(\Omega \times(0, T))}{\longrightarrow} f$ with $\left\|f_{n}\right\|_{L^{2}(\Omega \times(0, T))} \leqslant\|f\|_{L^{2}(\Omega \times(0, T))}$. 
Theorem 5. For each integer $n \geqslant 1$, there exists a unique function $u_{n}$ of the form (20) satisfying (21).

Proof. To solve the problem (21), we shall find

$$
p_{n}(t)=\left(p_{1 n}(t), p_{2 n}(t), \ldots, p_{n n}(t)\right) \in \mathbb{R}^{n}
$$

solution to

$$
\left\{\begin{array}{l}
j=1, \ldots, n \\
p_{j n}^{\prime \prime}+\sum_{k=1}^{n} \int_{\Omega \cup \Omega_{I}} \int_{\Omega \cup \Omega_{I}} \mathcal{D}^{*}\left(v_{j}\right) \cdot\left(\xi \cdot \mathcal{D}^{*}\left(v_{k}\right)\right) p_{k n}(t) d y d x=\int_{\Omega} f_{n} v_{j} d x, \\
p_{j n}(0)=\left(g, v_{j}\right)_{L^{2}(\Omega)}, \\
p_{j n}^{\prime}(0)=\left(h, v_{j}\right)_{L^{2}(\Omega)} .
\end{array}\right.
$$

According to standard existence theory for ODE, there exists a unique function

$$
p_{n}(t)=\left(p_{1, n}(t), p_{2, n}(t), \ldots, p_{n, n}(t)\right)
$$

satisfying (22) for a.e. $0 \leqslant t \leqslant T$.

\subsection{Energy estimates}

In order to show that $\left(u_{n}\right)_{n \geqslant 1}$ converges to a weak solution of (14), we will need some uniform estimates.

Theorem 6. There exists a positive constant $M$ such that

$$
\begin{aligned}
& \max _{0 \leqslant t \leqslant T}\left(\left\|u_{n}(t)\left|\|+\mid\| u_{n}^{\prime}(t) \|_{L^{2}\left(\Omega \cup \Omega_{I}\right)}\right)+\right\| u_{n}^{\prime \prime} \|_{L^{2}\left(0, T ; V_{c}^{*}\left(\Omega \cup \Omega_{I}\right)\right)}\right. \\
& \quad \leqslant M\left(\|f\|_{L^{2}\left(0, T ; L^{2}(\Omega)\right)}+\|g\|\|+\| h \|_{L^{2}(\Omega)}\right) \quad \text { for } n \geqslant 1 .
\end{aligned}
$$

Proof. We multiply equation $(21)$ by $p_{j n}^{\prime}(t)$, sum for $j=1, \ldots, n$, we find

$$
\left\langle u_{n}^{\prime \prime}, u_{n}^{\prime}\right\rangle_{V_{c}^{*}\left(\Omega \cup \Omega_{I}\right), V_{c}\left(\Omega \cup \Omega_{I}\right)}+\int_{\Omega \cup \Omega_{I}} \int_{\Omega \cup \Omega_{I}} \mathcal{D}^{*}\left(u_{n}\right) \cdot\left(\xi \cdot \mathcal{D}^{*}\left(u_{n}^{\prime}\right)\right) d y d x=\int_{\Omega} f_{n} u_{n}^{\prime} d x
$$

for a.e. $0 \leqslant t \leqslant T$.

which gives

$$
\begin{aligned}
\frac{d}{d t}\left(\left\|u_{n}^{\prime}\right\|_{L^{2}\left(\Omega \cup \Omega_{I}\right)}^{2}+\int_{\Omega \cup \Omega_{I}} \int_{\Omega \cup \Omega_{I}} \mathcal{D}^{*}\left(u_{n}\right) \cdot\left(\xi \cdot \mathcal{D}^{*}\left(u_{n}\right)\right) d y d x\right) & \leqslant 2\left\|f_{n}\right\|_{L^{2}(\Omega)}\left\|u_{n}^{\prime}\right\|_{L^{2}(\Omega)} \\
& \leqslant\left\|f_{n}\right\|_{L^{2}(\Omega)}^{2}+\left\|u_{n}^{\prime}\right\|_{L^{2}\left(\Omega \cup \Omega_{I}\right)}^{2}+\left\|u_{n}\right\| \|^{2} .
\end{aligned}
$$

Gronwall's inequality and the proposition (6) imply

$$
\begin{aligned}
\left\|u_{n}^{\prime}\right\|_{L^{2}\left(\Omega \cup \Omega_{I}\right)}^{2}+\|\| u_{n} \|\left.\right|^{2} & \leqslant M\left(\left\||| P_{n}(g) \mid\right\|^{2}+\left\|P_{n}(h)\right\|_{L^{2}\left(\Omega \cup \Omega_{I}\right)}^{2}+\left\|f_{n}\right\|_{L^{2}\left(0, T ; L^{2}(\Omega)\right)}\right) \\
& \leqslant M\left(\|g \mid\|^{2}+\|h\|_{L^{2}\left(\Omega \cup \Omega_{I}\right)}^{2}+\left\|f_{n}\right\|_{L^{2}\left(0, T ; L^{2}(\Omega)\right)}^{2}\right)
\end{aligned}
$$

as $0 \leqslant t \leqslant T$ was chosen arbitrarily, we obtain:

$$
\max _{0 \leqslant t \leqslant T}\left(\left\|u_{n}^{\prime}(t)\right\|_{L^{2}\left(\Omega \cup \Omega_{I}\right)}^{2}+\|\| u_{n} \|\left.\right|^{2}\right) \leqslant M\left(\||| g \mid\|^{2}+\|h\|_{L^{2}\left(\Omega \cup \Omega_{I}\right)}^{2}+\|f\|_{L^{2}\left(0, T ; L^{2}(\Omega)\right)}^{2}\right)
$$

To conclude, we fix any $\varphi \in V_{c}\left(\Omega \cup \Omega_{I}\right)$ with

$$
\|\varphi \mid\| \leqslant 1 \text { and } \varphi=P_{n}(\varphi)+\psi
$$

Mathematical Modeling and Computing, Vol. 7, No. 2, pp. 334-344 (2020) 
where $\left(\psi, v_{j}\right)_{L^{2}\left(\Omega \cup \Omega_{I}\right)}=0$ for $1 \leqslant j \leqslant n$. We get:

$$
\left\langle u_{n}^{\prime \prime}, \varphi\right\rangle_{V_{c}^{*}\left(\Omega \cup \Omega_{I}\right), V_{c}\left(\Omega \cup \Omega_{I}\right)}=\int_{\Omega} f_{n} P_{n}(\varphi) d x-\int_{\Omega \cup \Omega_{I}} \int_{\Omega \cup \Omega_{I}} \mathcal{D}^{*}\left(u_{n}\right) \cdot\left(\xi \cdot \mathcal{D}^{*}\left(P_{n}(\varphi)\right)\right) d y d x .
$$

Consequently, since $\left\|\left|P_{n}(\varphi)\right|\right\| \leqslant 1$

$$
\left|\left\langle u_{n}^{\prime \prime}, \varphi\right\rangle\right|_{V_{c}^{*}\left(\Omega \cup \Omega_{I}\right), V_{c}\left(\Omega \cup \Omega_{I}\right)} \leqslant M\left(\left\|f_{n}\right\|_{L^{2}(\Omega)}^{2}+\left|\| u_{n}\right|||\right),
$$

finally, using (3.3) we get

$$
\left\|u_{n}^{\prime \prime}\right\|_{L^{2}\left(0, T ; V_{c}^{*}\left(\Omega \cup \Omega_{I}\right)\right)} \leqslant M\left(\|f\|_{L^{2}\left(0, T ; L^{2}(\Omega)\right)}^{2}+\|\| g\|\|^{2}+\|h\|_{L^{2}(\Omega)}^{2}\right) .
$$

\subsection{Existence and uniqueness result}

Theorem 7. The nonlocal hyperbolic problem (14) admits a unique weak solution.

Proof. Using the previous Theorem 6, we conclude that $\left(u_{n}\right)_{n \geqslant 1}$ is bounded in $L^{2}\left(0, T ; V_{c}\left(\Omega \cup \Omega_{I}\right)\right)$, with $\left(u_{n}^{\prime}\right)_{n \geqslant 1}$ is bounded in $L^{2}\left(0, T ; L^{2}\left(\Omega \cup \Omega_{I}\right)\right)$, and $\left(u_{n}^{\prime \prime}\right)_{n \geqslant 1}$ is bounded in $L^{2}\left(0, T ; V_{c}^{*}\left(\Omega \cup \Omega_{I}\right)\right)$.

Consequently, there exists a subsequence still denoted $\left(u_{n}\right)_{n \geqslant 1}$ and a function $u \in L^{2}\left(0, T ; V_{c}(\Omega \cup\right.$ $\left.\left.\Omega_{I}\right)\right)$ with $u^{\prime} \in L^{2}\left(0, T ; L^{2}\left(\Omega \cup \Omega_{I}\right)\right)$ and $u^{\prime \prime} \in L^{2}\left(0, T ; V_{c}^{*}\left(\Omega \cup \Omega_{I}\right)\right)$, such that:

$$
\left\{\begin{array}{lll}
u_{n} \rightarrow u & \text { in } \quad L^{2}\left(0, T ; V_{c}\left(\Omega \cup \Omega_{I}\right),\right. \\
u_{n}^{\prime} \rightarrow u^{\prime} & \text { in } \quad L^{2}\left(0, T ; L^{2}\left(\Omega \cup \Omega_{I}\right),\right. \\
u_{n}^{\prime \prime} \rightarrow u^{\prime \prime} & \text { in } \quad L^{2}\left(0, T ; V_{c}^{*}\left(\Omega \cup \Omega_{I}\right),\right.
\end{array}\right.
$$

next, fix an integer $N$ and select $n \geqslant N$, choose a function $\psi \in L^{2}(0, T)$ and $\varphi \in V_{c}\left(\Omega \cup \Omega_{I}\right)$. We multiply $(21)$ by $P_{n}(\varphi) \psi$, sum $j=1, \ldots, N$ and integrate with respect to $t$ to discover:

$$
\begin{array}{r}
\int_{0}^{T}\left(\left\langle u_{n}^{\prime \prime}, P_{n}(\varphi) \psi(t)\right\rangle_{V_{c}^{*}\left(\Omega \cup \Omega_{I}\right), V_{c}\left(\Omega \cup \Omega_{I}\right)}+\int_{\Omega \cup \Omega_{I}} \int_{\Omega \cup \Omega_{I}} \mathcal{D}^{*}\left(u_{n}\right) \cdot\left(\xi \cdot \mathcal{D}^{*}\left(P_{n}(\varphi) \psi\right)\right) d y d x\right) d t \\
=\int_{0}^{T} \int_{\Omega} f_{n} P_{n}(\varphi) \psi d x d t
\end{array}
$$

by passing to weak limits, together with the fact that $P_{n}(\varphi) \stackrel{V_{c}\left(\Omega \cup \Omega_{I}\right)}{\longrightarrow} \varphi$ we obtain:

$$
\begin{aligned}
\int_{0}^{T}\left(\left\langle u^{\prime \prime}, \varphi\right\rangle_{V_{c}^{*}\left(\Omega \cup \Omega_{I}\right), V_{c}\left(\Omega \cup \Omega_{I}\right)} \psi(t)+\int_{\Omega \cup \Omega_{I}} \int_{\Omega \cup \Omega_{I}} \mathcal{D}^{*}(u) \cdot\left(\xi \cdot \mathcal{D}^{*}(\varphi \psi(t))\right) d y d x\right) d t & =\int_{0}^{T} \int_{\Omega} f \varphi \psi(t) d x d t
\end{aligned}
$$

for all $\psi \in L^{2}(0, T)$ and $\varphi \in V_{c}\left(\Omega \cup \Omega_{I}\right)$. This terminates the proof.

It remains to prove that $u(0)=g$ and $u^{\prime}(0)=h$. For this purpose, we choose any function $\varphi \in V_{c}\left(\Omega \cup \Omega_{I}\right)$ and $\psi \in \mathcal{C}^{1}([0, T])$ such that $\psi(T)=\psi^{\prime}(T)=0$. Integrating by parts twice with respect to $t$ in (26) yields:

$$
\begin{aligned}
\int_{0}^{T}\left(\int_{\Omega \cup \Omega_{I}} u \varphi \psi^{\prime \prime}(t) d x+\int_{\Omega \cup \Omega_{I}}\right. & \left.\int_{\Omega \cup \Omega_{I}} \mathcal{D}^{*}(u) \cdot\left(\xi \cdot \mathcal{D}^{*}(\varphi \psi(t))\right) d y d x\right) d t \\
& =\int_{0}^{T} \int_{\Omega} f \varphi \psi(t) d x d t-\int_{\Omega} u(0) \varphi \psi^{\prime}(0) d x+\int_{\Omega} u^{\prime}(0) \varphi \psi(0) d x
\end{aligned}
$$

Mathematical Modeling and Computing, Vol.7, No. 2, pp. 334-344 (2020) 
Similarly from (25) we get:

$$
\begin{aligned}
\int_{0}^{T}\left(\int_{\Omega \cup \Omega_{I}} u_{n} P_{n}(\varphi) \psi^{\prime \prime}(t) d x+\int_{\Omega \cup \Omega_{I}} \int_{\Omega \cup \Omega_{I}} \mathcal{D}^{*}\left(u_{n}\right) \cdot\left(\xi \cdot \mathcal{D}^{*}\left(P_{n}(\varphi) \psi(t)\right)\right) d y d x\right) d t \\
=\int_{0}^{T} \int_{\Omega} f_{n} P_{n}(\varphi) \psi(t) d x d t-\int_{\Omega} u_{n}(0) P_{n}(\varphi) \psi^{\prime}(0) d x+\int_{\Omega} u_{n}^{\prime}(0) P_{n}(\varphi) \psi(0) d x
\end{aligned}
$$

by passing to the limit, we obtain:

$$
\begin{aligned}
\int_{0}^{T}\left(\int_{\Omega \cup \Omega_{I}} u \varphi \psi^{\prime \prime}(t) d x+\int_{\Omega \cup \Omega_{I}} \int_{\Omega \cup \Omega_{I}}\right. & \left.\mathcal{D}^{*}(u) \cdot\left(\xi \cdot \mathcal{D}^{*}(\varphi \psi(t))\right) d y d x\right) d t \\
& =\int_{0}^{T} \int_{\Omega} f \varphi \psi(t) d x d t-\int_{\Omega} g \varphi \psi^{\prime}(0) d x+\int_{\Omega} h \varphi \psi(0) d x
\end{aligned}
$$

comparing those results, we conclude that $u(0)=g$ and $u^{\prime}=h$.

Finally, we announce the uniqueness of the weak solution to (14).

Theorem 8. A weak solution of (14) is unique.

Proof. Since the equation is linear, to show uniqueness it is sufficient to show that the only solution $u$ of (14) with zero data $f=g=h=0$ is $u=0$.

To verify this, fix $0 \leqslant s \leqslant T$ and set

$$
\varphi(t)= \begin{cases}\int_{t}^{s} u(\tau) d \tau & \text { if } \quad 0 \leqslant t<s \\ 0 & \text { if } \quad s \leqslant t \leqslant T\end{cases}
$$

Then $\varphi(t) \in V_{c}\left(\Omega \cup \Omega_{I}\right)$ for each $0 \leqslant t \leqslant T$, which allows us to write

$$
\int_{0}^{s}\left(\left\langle u^{\prime \prime}, \varphi\right\rangle_{V_{c}^{*}\left(\Omega \cup \Omega_{I}\right), V_{c}\left(\Omega \cup \Omega_{I}\right)}+\int_{\Omega \cup \Omega_{I}} \int_{\Omega \cup \Omega_{I}} \mathcal{D}^{*}(u) \cdot\left(\xi \cdot \mathcal{D}^{*}(\varphi)\right) d y d x\right) d t=0,
$$

since $u^{\prime}=0$ and $\varphi(s)=0$ by integrating by parts, we obtain:

$$
\int_{0}^{s}\left(-\int_{\Omega \cup \Omega_{I}} u^{\prime} \varphi^{\prime} d x+\int_{\Omega \cup \Omega_{I}} \int_{\Omega \cup \Omega_{I}} \mathcal{D}^{*}(u) \cdot\left(\xi \cdot \mathcal{D}^{*}(\varphi)\right) d y d x\right) d t=0,
$$

now as $\varphi^{\prime}=-u(0 \leqslant t \leqslant s)$, we obtain:

$$
\int_{0}^{s}\left(\int_{\Omega \cup \Omega_{I}} u^{\prime} u d x-\int_{\Omega \cup \Omega_{I}} \int_{\Omega \cup \Omega_{I}} \mathcal{D}^{*}\left(\varphi^{\prime}\right) \cdot\left(\xi \cdot \mathcal{D}^{*}(\varphi)\right) d y d x\right) d t=0,
$$

then

$$
\int_{0}^{s} \frac{d}{d t}\left(\|u\|_{L^{2}\left(\Omega \cup \Omega_{I}\right)}^{2}-\int_{\Omega \cup \Omega_{I}} \int_{\Omega \cup \Omega_{I}} \mathcal{D}^{*}(\varphi) \cdot\left(\xi \cdot \mathcal{D}^{*}(\varphi)\right) d y d x\right) d t=0
$$

here

$$
\|u(s)\|_{L^{2}\left(\Omega \cup \Omega_{I}\right)}^{2}+\|\varphi(0)\| \|=0
$$

Consequently $u=0$ on $[0, T]$.

[1] Du Q., Gunzburger M., Lehoucq R. B., Zhou K. A nonlocal vector calculus, nonlocal volume-constrained problems, and nonlocal balance laws. Mathematical Models and Methods in Applied Sciences. 23 (03), 493-540 (2013).

Mathematical Modeling and Computing, Vol. 7, No. 2, pp. 334-344 (2020) 
[2] Gilboa G., Osher S. Nonlocal operators with applications to image processing. Multiscale Model. Simul. 7 (3), 1005-1028 (2008).

[3] Guan Q., Gunzburger M. Analysis and approximation of a nonlocal obstacle problem. J. Comput. Appl. Math. 313, 102-118 (2017).

[4] Gunzburger M., Lehoucq R. B. A nonlocal vector calculus with application to nonlocal boundary value problems. Multiscale Model. Simul. 8 (5), 1581-1598 (2010).

[5] Kindermann S., Osher S., Jones P. W. Deblurring and denoising of images by nonlocal functionals. Multiscale Model. Simul. 4 (4), 1091-1115 (2005).

[6] Alberti G., Bellettini G. A nonlocal anisotropic model for phase transitions. I. The optimal profile problem. Mathematische Annalen. 310, 527-560 (1998).

[7] Bates P.W., Chmaj A. An integrodifferential model for phase transitions: stationary solutions in higher space dimensions. J. Stat. Phys. 95 (5/6), 1119-1139 (1999).

[8] Rosasco L., Belkin M., De Vito E. On learning with integral operators. J. Mach. Learn. Res. 11, 905-934 (2010).

[9] Du Q., Gunzburger M., Lehoucq R. B., Zhou K. Analysis and approximation of nonlocal diffusion problems with volume constraints. SIAM Rev. 54 (4), 667-696 (2012).

[10] Zeidler E. II/A: Linear Monotone Operators. Nonlinear Functional Analysis and Its Applications. SpringerVerlag, New York (1990).

\title{
Аналіз задачі нелокального поширення хвиль з об'ємними обмеженнями
}

\author{
Айт Белла Ф. 3. ${ }^{1}$, Ель-Рабі М. ${ }^{2}$, Хакім А. ${ }^{1}$, Лагріб А. ${ }^{3}$ \\ ${ }^{1}$ Університет Каді Айяд, \\ проспект Абделькрим Хаттабі, Марракеш, 40000, Марокко \\ 2 Політехнічний інститут UniLaSalle, \\ вул. Ду Тронке, 3, Мон-Сент-Енъян, 76130, Франція \\ ${ }^{3}$ Університет Султана Мулай Сліман, \\ Авеню Ібн Халдун, Бені-Меллал, 23000, Марокко
}

\begin{abstract}
У даній статті розробляється модель нелокального поширення, яка описує дифузійний хвильовий процес. Основна мотивація цієї роботи - це застосування нелокального векторного числення, яке введене та розвинене Ду та ін. [1], до такої гіперболічної задачі. Крім того, використовуючи функцію густини, деякі апріорні оцінки і підхід Гальоркіна, ми доводимо існування та єдиність слабкого розв'язку нелокального хвильового рівняння, яке має широке застосування.
\end{abstract}

Ключові слова: наближення Гальоркіна, нелокальні оператори, нелокальне векторне числення, задача з обмеженнями на об'єм, хвильове рівняння. 